\title{
AVANCES DE LA INVESTIGACIÓN SOBRE LA GESTIÓN INTEGRAL DEL RECURSO HIDRICO EN COLOMBIA
}

\section{RESEARCH ADVANCES ON THE INTEGRAL MANAGEMENT OF WATER RESOURCE IN COLOMBIA}

\author{
Sandra Marisol Hernández Pasichana ${ }^{1}$, Adriana Posada Arrubla ${ }^{2}$ \\ ${ }^{1}$ Ingeniera Ambiental, M.Sc. Ciencias Ambientales. Universidad de Ciencias Aplicadas y Ambientales U.D.C.A, Calle 222 \\ No. 55-37 Bogotá, Colombia, e-mail: smhernandezp@gmail.com; iDhttps://orcid.org/0000-0002-9794-1530; ${ }^{2}$ Economista \\ Agrícola, Esp. Gestión Social y Ambiental, M.Sc. Planeación Urbana y Regional, Docente-Investigadora Facultad Ingenierías. \\ Universidad de Ciencias Aplicadas y Ambientales U.D.C.A, Calle 222 No. 55-37, Bogotá D.C., Colombia, e-mail: aposada@ \\ udca.edu.co; (iDhttps://orcid.org/0000-0002-2440-0805
}

\author{
Rev. U.D.C.A Act. \& Div. Cient. 21(1):553-563, Julio-Diciembre, 2018 \\ https://doi.org/10.31910/rudca.v21.n2.2018.1079
}

Artículo de acceso abierto publicado por Revista U.D.C.A Actualidad \& Divulgación Científica

bajo una licencia Creative Commons CC BY-NC 4.0

\section{RESUMEN}

Por la relevancia ambiental, económica y social del recurso hídrico es importante contar con una investigación pertinente, que sirva de base para su gestión integral, por lo cual, se quiere resaltar, los avances de la comunidad científica de Colombia, que realiza investigación sobre la gestión integral del recurso hídrico. Se utilizó metodología exploratoria documental y de la plataforma Scienti y se efectuó indagación en grupos focales, regional y nacional. Se identificaron seis líneas y 37 sublíneas, en el sector público, a nivel nacional; 166 grupos de investigación, profundizando en cinco temáticas, que desarrollan en su interior 54 líneas de investigación y, en el ámbito regional, se detectó una brecha entre lo que se ha logrado hacer y un listado de asuntos pendientes por investigar.

Palabras clave: políticas públicas y agua, retos agua, temáticas agua.

\section{ABSTRACT}

Because of the environmental, economic and social importance of water resources, it is important to have a relevant research, that serves as a base for its integral management. Therefore, it is intended to highlight the advances of the scientific community of Colombia, carrying out research on the integrated management of water resources. Documentary and platform Scienti exploratory methodology was used and inquiry took place in focus groups, at national and regional level. Six lines and 37 sub-lines were identified in the public sector at the national level; 166 research groups, deepening into five thematic themes, which developed in its interior 54 research lines; and at the regional level, a gap between what has been achieved and a listing of issues to investigate was found.

Keywords: public policies and water, water challenges, themed water.

\section{INTRODUCCIÓN}

La investigación ambiental comprende diversidad de temáticas, entre las que se encuentra, el recurso hídrico y su correspondiente gestión integral. Según Hassan (2011), los principales paradigmas en la historia de la gestión del agua han sido: el espiritual-religioso, el estético-recreacional, el científico, el ecológico, el de hidráulica e ingeniería, el económico-financiero, el gerencial y de gobernanza y el ético-legal. Y, aunque en el mundo los asuntos asociados a la gestión integral del recurso hídrico (GIRH) llevan más de 50 años, según Winz et al. (2009), la investigación científica al respecto, todavía tiene mucho camino por recorrer.

Después de más de cinco décadas de investigación, el WWAP (2018) alerta sobre una demanda mundial de agua, que ha aumentado a un ritmo del $1 \%$ anual, en función del aumento de población, del desarrollo económico y de los cambios en los patrones de consumo, entre otros factores. 
Por ello, el reto ambiental sobre la gestión del agua, incluye, poder lograr un balance entre las prioridades de crecimiento económico, disminución de la pobreza y conservación del recurso, pero se sigue tratando el tema de manera independiente a la gestión de los ecosistemas, lo cual, ha conducido a la promoción de otro tipo de enfoques y de visiones, que buscan abordar esta temática, de una manera integral, reconociendo el papel que cumple el agua en los ecosistemas y en asociar su gestión a su manejo, con una perspectiva integral (Andrade \& Le Blas, 2004).

Para Pahl-Wostl (2007), la tradición en la gestión de los recursos naturales, se ha hecho bajo el esquema del control, pero para la complejidad ambiental, se requiere un enfoque de manejo adaptado a cada circunstancia, donde la sociedad juega un papel relevante y, es por ello, que la comunidad científica debe replantear cambios de enfoques y de acciones inter y transdisciplinarias. En este punto, Martínez et al. (2014) especifican que la GIRH es una nueva forma de enmarcar la gestión del agua, en conjunto con otras perspectivas, que pueden ser utópicas, si se generalizan demasiado o podrían ser un gran desafío, si se integran a realidades tangibles.

La Gestión Integral del Recurso Hídrico, se define como un proceso que promueve el manejo y el desarrollo coordinado del agua, la tierra y demás recursos, con el fin de maximizar el bienestar social y económico, de manera equitativa y sin comprometer la sustentabilidad de los ecosistemas (GWP, 2000). La GIRH ha sido aceptada internacionalmente, como el camino hacia un desarrollo y gestión eficiente, equitativa y sostenible, de unos recursos hídricos cada vez más limitados, que permita abordar unas demandas en competición (UN Water, 2008).

En Colombia, la comunidad científica que dedica sus investigaciones en relación con la GIRH, ha adelantado un trabajo de suma importancia. Aun así, estos esfuerzos no han logrado atender la diversidad de problemáticas existentes al respecto, ni tampoco influir lo suficiente, para que sea considerada una base científica en la toma de decisiones del país, a lo cual, se suma que, las diferentes necesidades investigativas de una región a otra, corresponden a particularidades distintas. La GIRH es importante como referente para el diseño de modelos de gestión pública del agua (MAVDT, 2010) y, además, los avances en su investigación generan desarrollo a todo nivel (República de Colombia, 2001), razón por la cual, el país empezó a establecer directrices normativas de fomento a la investigación científica y al desarrollo tecnológico, para soportar decisiones ambientales y del recurso hídrico (Congreso de Colombia,1990).
Se resalta en el país un proceso que ha sido desarrollado simultáneamente, aunque no de manera articulada, entre dos sistemas: el Sistema Nacional Ambiental (SINA), encabezado por el Ministerio de Ambiente y Desarrollo sostenible (MADS) y el Sistema Nacional de Ciencia Competitividad, Tecnología e innovación (SNCCTI), liderado por Colciencias (Sáenz, 2003). Estos sistemas han impulsado diferentes políticas y planes y se integran a cinco institutos de investigación, vinculados y adscritos al MADS: Instituto de hidrología, Meteorología y Estudios Ambientales (IDEAM), Instituto de Investigaciones Marinas y Costeras José Benito Vives de Andreis -(INVEMAR), Instituto de Investigación de Recursos Biológicos Alexander von Humboldt e Instituto de Investigaciones Ambientales del Pacífico John Von Neumann e Instituto Amazónico de Investigaciones Científicas (SINCHI), los cuales, en conjunto, han avanzado en relación con el plan estrategico nacional de investigación ambiental (MAVDT \& QUINAXI, 2007).

Para Guhl \& Leyva (2015), estos institutos de investigación están muy centrados en las ciencias naturales, en especial, en las biológicas y en la investigación básica y consideran que se debe avanzar en realizar proyectos holísticos, con enfoques complejos y en la formación de equipos interdisciplinarios, en busca del aprovechamiento sostenible de los sistemas naturales, en interacción con los sistemas sociales. Lo que coincide con el planteamiento de Martínez et al. (2014), para quienes, frente a los desafíos contemporáneos de la GIRH, se esperan respuestas sobre la demanda y la disponibilidad del agua, en especial, de las políticas orientadas a estimular el desarrollo y el despliegue de tecnologías y de enfoques innovadores.

La investigación, según De Barrera (2010), es considerada como uno de los procesos más importantes en la construcción de conocimiento y las líneas de investigación cumplen con el propósito de organizar y orientar este proceso, para contribuir a dinamizar la actividad investigativa y su gestión. Respecto a las líneas de investigación, Agudelo (2004) destaca el proceso de construcción colectiva de personas e investigadores, que aportan al conocimiento, con proyectos interdisciplinarios, que se articulan entre sí.

Motivado por lo anterior, se ha establecido, como objetivo de este artículo, compartir la metodología y sus respectivos resultados de una investigación, que resalta los avances de la comunidad científica de Colombia que, a su vez, realiza investigación sobre la gestión integral del recurso hídrico (GIRH). En pocas palabras, en este artículo, se muestra una investigación sobre varias investigaciones. 


\section{MATERIALES Y MÉTODOS}

La metodología combinó, por una parte, la recolección y el análisis de información secundaria de los actores estratégicos asociados a los dos sistemas más representativos en Colombia, sobre la GIRH: SINA y SNCCTI y, por otra, la obtención de información primaria, adquirida en reuniones específicas, que se llevaron a cabo, utilizando el prototipo de grupos focales, ya que brindan la posibilidad de interactuar con el grupo objetivo o grupo meta (Ivankovich \& Araya, 2011). La mezcla de ambas formas de indagación permitió desarrollar tres etapas en el proceso investigativo (Figura 1).

Etapa Uno: Exploración de índole nacional, de corte público. Con el apoyo del MADS, se realizaron consulta a expertos de la dirección de gestión integral de recurso hídrico, a partir de las siguientes dos preguntas: ¿Qué líneas de investigación relacionadas con la GIRH, se han desarrollado?, ¿Qué líneas de investigación, a nivel nacional, se deben trabajar para una GIRH?

Esta actividad, se complementó con la revisión de los planes Institucionales de investigación ambiental 2015-2018, correspondientes a los cinco institutos vinculados y adscritos: IDEAM, INVEMAR, Instituto Alexander von Humboldt e Instituto John Von Neumann y SINCHI.

Etapa dos: Exploración de índole nacional, de corte científico. La base de datos utilizada en esta etapa de la indagación fue la suministrada por Colciencias, a través de su plataforma Scienti, entre septiembre y octubre de 2017, momento en el cual, no existía una agregación de la informa-

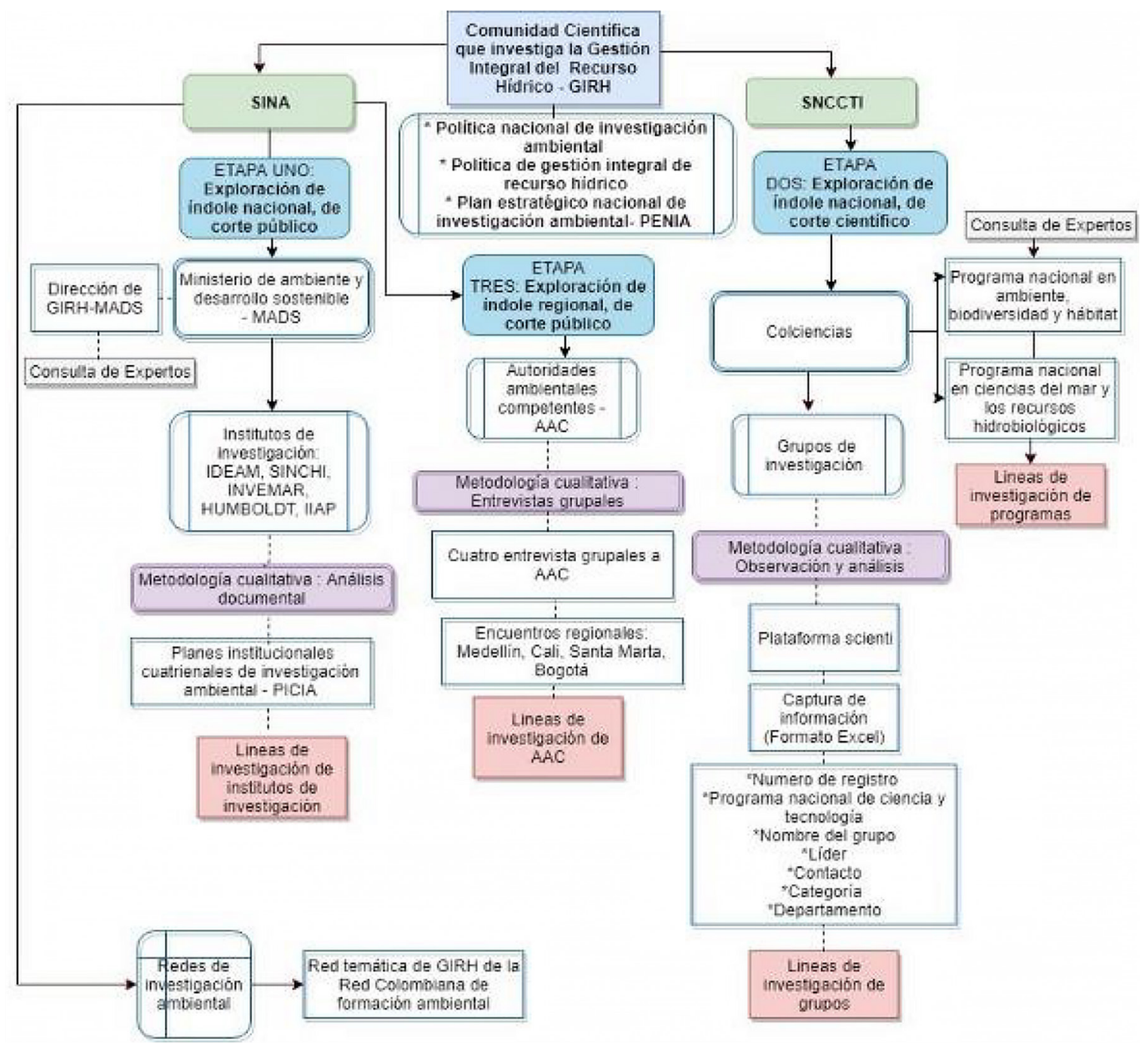

Figura 1. Metodología para la identificación de líneas de investigación sobre GIRH, por grupos de actores estratégicos. 
ción similar a la que se logró consolidar en este proyecto. Al acceder minuciosamente a esta plataforma, se logró identificar a los grupos de investigación relacionados con la GIRH y se capturó la información en formato Excel, con los siguientes aspectos particulares: nombre del grupo de investigación, líder, contacto, categoría, entidad territorial, líneas investigación.

Etapa tres: Exploración de índole regional, de corte público. Con el apoyo y la coordinación del MADS, se realizaron cuatro encuentros regionales, en ciudades centrales de Colombia, en donde se logró agrupar a las 42 Autoridades ambientales, según centros de encuentro tradicional o cercanía geográfica. El desarrollo de estos talleres en grupos focales, se llevó a cabo, a partir de las siguientes preguntas: ¿La entidad, a la cual pertenece, ha realizado o participado en proyectos de investigación relacionados con la GIRH?, ¿Qué temas de Investigación considera la entidad son de relevancia para la GIRH?

Estos grupos focales, en la etapa tres, se organizaron con la participación de funcionarios de cuatro diferentes regiones, que representan a la mayoría del territorio colombiano, con la intervención de funcionarios conocedores del tema, al servicio de las siguientes instituciones, así:

Grupo 1: Región Occidente. Taller realizado en Medellín, con 15 participantes, de AMVA, PNN, CORNARE, MADS.

Grupo 2: Región Sur. Taller realizado en Cali, con 14 participantes, de CVC, PNN, DAGMA, CRQ, MADS.

Grupo 3: Región Centro. Taller realizado en Bogotá, con 20 participantes, de CORTOLIMA, CAM, CAR, CORPOGUAVIO, CORPONOR, CORPOBOYACA, CAS, CDMB, PNN, ANLA, CORPOCHIVOR, MADS.

Grupo 4: Región Norte. Taller realizado en Santa Marta, con 12 participantes de CORPAMAG, DADMA, CORPOCALDAS, CORPOGUAJIRA, CRA, CARDIQUE, PNN, MADS.

\section{RESULTADOS Y DISCUSIÓN}

En relación directa con el desarrollo de las actividades realizadas en las tres etapas metodológicas, se logró establecer la siguiente situación, respecto al perfil de la investigación sobre la GIRH, en Colombia.

Resultados de la exploración de índole nacional, de corte público. En el cuadro 1, se sintetizan las seis líneas de investigación identificadas, como aquellas que se deberían trabajar para una GIRH y, para cada una de ellas, varios temas internos, que corresponden a 37 sublíneas, perfiladas desde la función pública nacional, como las necesarias para investigar al detalle.
Se encontró, que los temas ya no se abordan de manera particular, sino combinados, especialmente, se integra lo relacionado con la gestión y la gobernanza, como asuntos emergentes. Frente, a lo cual, las autoras consideran que, entre las instancias del gobierno Nacional urge una integración de los alcances, objetivos, métodos y estrategias, para investigar sobre el agua, pero no de manera fragmentada, sino como un todo, en el que se pueda diferenciar sus partes, para asumir, a profundidad, las investigaciones, evitando así superposiciones e investigaciones repetitivas.

Resultados de la exploración de índole nacional, de corte científico. En el cuadro 2, se presenta el trabajo realizado por los grupos de investigación clasificados por Colciencias, a octubre 2017, de manera agrupada por temas o áreas de conocimiento, en cinco bloques. Al interior de estos cinco temas, se encontraron 54 líneas de Investigación, relacionadas con GIRH.

Para lograr este resultado, se revisaron 166 grupos, que corresponden al 2\%, de los 9.013 grupos de investigación en general, que figuran en la plataforma Scienti, representados en orden descendente para este caso, en los programas de: ambiente, biodiversidad y hábitat, con 85 grupos; ciencias del mar y los recursos hidrobiológicos, con 25 grupos; ciencias básicas, con 23 grupos; ciencia, tecnología e innovación en ciencias agropecuarias, 12 grupos; ciencia, tecnología e innovación en ingeniería, 8 grupos; investigación en energía y minería, 4 grupos; ciencia, tecnología e innovación en geociencias, 3 grupos; ciencia, tecnología e innovación en ciencias humanas, sociales y educación, 2 grupos; ciencia tecnología e innovación en salud, 2 grupos y biotecnología, 2 grupos.

De estos 166 grupos que realizan investigaciones relacionadas con la GIRH, el $30 \%$ corresponde a la más alta categoría de investigación, seguidos, del $20 \%$, en categoría avanzada; un $37 \%$, en categoría buena, un $10 \%$, en categoría en despegue y un $3 \%$, que aún no tiene clasificación. En cuanto a la ubicación regional, 42 grupos, se encuentran en Bogotá, seguido de Antioquia, con 24 y el Valle del Cauca, 16 grupos. Para el caso de las universidades son la de carácter público las que más se destacan con este conteo: la Universidad Nacional de Colombia, con 22 grupos; la Universidad de Antioquia, con 10 y la Universidad del Valle, con 9 grupos.

En esta exploración, se encontró que, en Colombia, si bien hay un buen número de grupos dedicados a la investigación en relación con el agua, también es numeroso el cambio de enfoques, debido, en parte, a que el recurso, factor, componente o subsistema, se estudia en complemento con otros temas. Lo que para las autoras es un potencial, pero se podría maximizar, si entre los diversos grupos de investigación, se generan redes, que ayuden a perfilar, no solo la interdisci- 
Cuadro 1. Posibles líneas y sublíneas de investigación, para la GIRH, perfiladas desde la óptica nacional, de corte público.

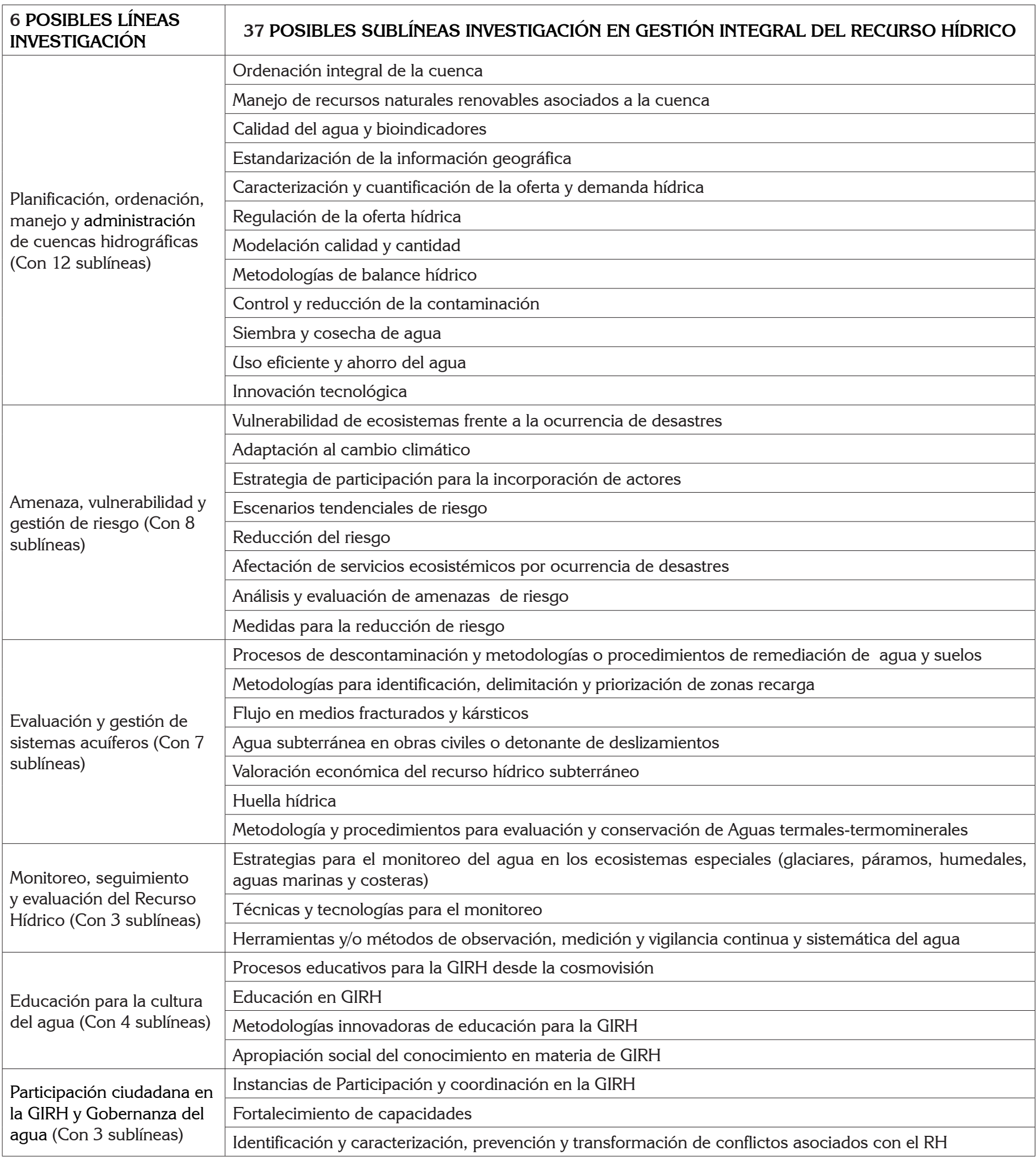


Cuadro 2. Temas y líneas de investigación relacionadas con GIRH, según grupos de investigación, clasificados en Colciencias, a octubre 2017.

\begin{tabular}{|c|c|c|c|c|}
\hline $\begin{array}{l}\text { Biodiversidad y servicios } \\
\text { ecosistémicos (\#12) }\end{array}$ & $\begin{array}{l}\text { Impactos, tratamiento } \\
\text { y calidad (\#12) }\end{array}$ & $\begin{array}{r}\text { Procesos y } \\
(\#)\end{array}$ & $\begin{array}{l}\text { Gestión del } \\
\text { riesgo y } \\
\text { saneamiento } \\
(\# 5)\end{array}$ & $\begin{array}{c}\text { Gestión ambiental } \\
\text { (\#11) }\end{array}$ \\
\hline 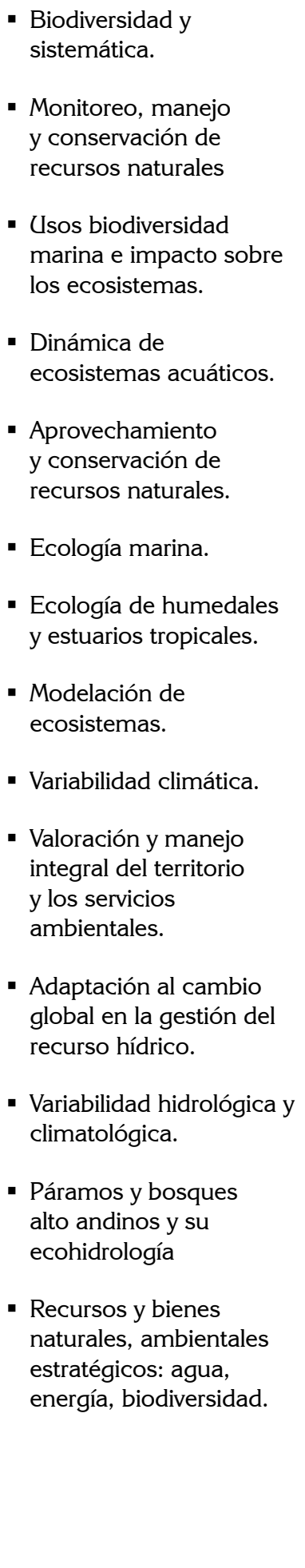 & $\begin{array}{l}\text { - Evaluación niveles } \\
\text { de traza de } \\
\text { contaminantes. } \\
\text { - Técnicas de análisis } \\
\text { químico. } \\
\text { - Calidad del agua en } \\
\text { corrientes hídricas. } \\
\text { - Bioindicación. } \\
\text { - Tratamiento aguas. } \\
\text { - Biorremediación } \\
\text { aguas y recuperación } \\
\text { de suelos. } \\
\text { de aguas con energía } \\
\text { solar. } \\
\text { - Calidad física, química } \\
\text { y biológica del agua. } \\
\text { recursos hídricos. } \\
\text { - Evaluación de impacto } \\
\text { ambiental en recursos } \\
\text { hidrobiológicos } \\
\text { continentales. } \\
\text { - Análisis de suelos y } \\
\text { aguas, y tecnologías } \\
\text { descontaminación. } \\
\text { - Tratamiento y control } \\
\text { de aguas residuales. } \\
\text { la remocionosión de } \\
\text { contaminantes en } \\
\text { agua, remoción de } \\
\text { metales pesados y } \\
\text { compuestos orgánicos } \\
\text { - } \\
\text { - }\end{array}$ & 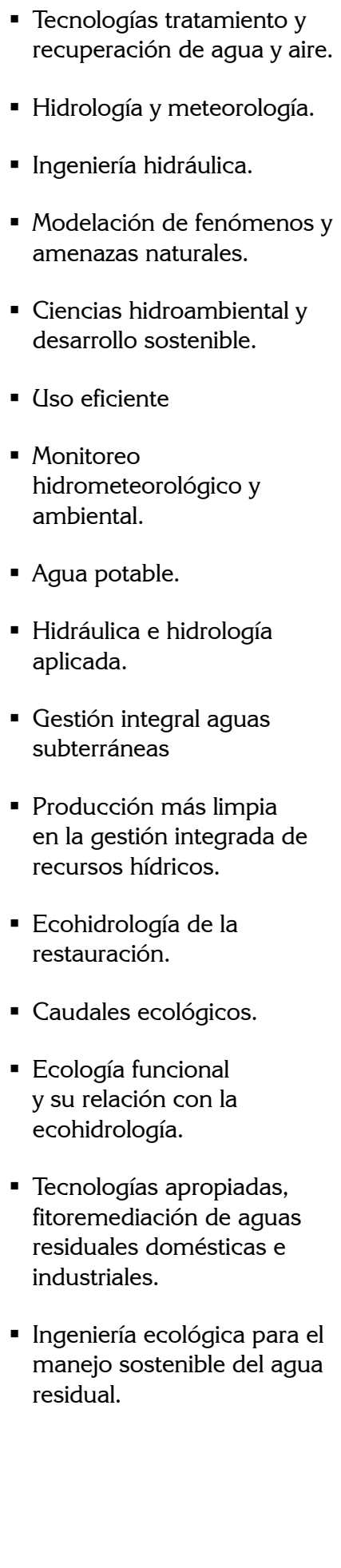 & $\begin{array}{l}\text { - Gestión del } \\
\text { riesgo por } \\
\text { desastres } \\
\text { naturales o } \\
\text { antrópicos. } \\
\text { - Saneamiento } \\
\text { básico y gestión } \\
\text { ambiental. } \\
\text { - Gestión del } \\
\text { riesgo de } \\
\text { desastres. } \\
\text { - Agua, } \\
\text { saneamiento } \\
\text { ambiental } \\
\text { y servicios } \\
\text { públicos } \\
\text { domiciliarios. } \\
\text { - Gestión del } \\
\text { riesgo por } \\
\text { inundación } \\
\text { Soluciones } \\
\text { ecoeficientes } \\
\text { para la } \\
\text { preservación } \\
\text { de las fuentes } \\
\text { hídricas. }\end{array}$ & 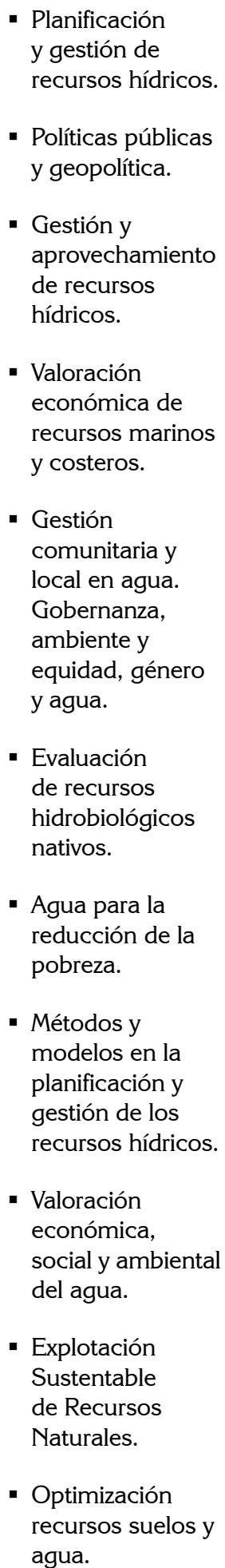 \\
\hline
\end{tabular}


plina, sino el diálogo de saberes con las comunidades y los sectores privados.

Resultados de la exploración de índole regional, de corte público. Respecto a la investigación pública, de nivel regional, en los cuadros 3 y 4, se logró recabar la información sobre el avance de la investigación en GIRH y las sugerencias de los temas que se deben abordar, resultante del trabajo con los 4 grupos focales regionales.

En cuanto a los avances regionales sobre la investigación en GIRH, en Colombia, se observan resultados bastante ligados a la normativa que, en su momento, establece el gobierno de turno, cuyos resultados están en relación directa con la disponibilidad técnica y financiera, para cada caso. Y si se compara con las propuestas planteadas en los grupos focales, se observa un numeroso e importante acervo de ideas evolucionadas, exigentes, con conocimiento de causa, visionarias y diversas, más no se le otorga un énfasis significativo a la gobernanza del agua.

Con lo anteriormente expuesto y a la luz del objetivo de este artículo, resaltar los avances de la comunidad científica de Colombia que realiza investigación sobre la GIRH, se evidencia que hay un buen camino recorrido, a nivel nacional y regional, pero que es más extenso, el trayecto que falta por recorrer, ya que, al comparar avances, contra propuestas de un deber ser, es más copiosa la lista de asuntos pendientes. También, se puede contrastar la relación exacta o dispersa de las líneas y sublíneas de investigación, frente a la GIRH, lo que evidencia una cantidad de temas fragmentados, que requieren ópticas más integrales, no solo de enfoques técnicos sobre el sistema, sino de lo que posibilita el diálogo de saberes con las comunidades.

De esta investigación se deduce, por una parte, que, al tener un mayor conocimiento sobre la GIRH, las oportunidades de progreso para el país se amplían y se pueden proyectar escenarios para el beneficio de todos los implicados, pero, por otra, se observa un vacío en la investigación y en las acciones sobre la gobernanza. Un estudio, como el aquí presentado, no es suficiente para identificar con precisión, el camino a seguir respecto a la GIRH, debido a que aborda los niveles nacional y regional, desde lo público y lo científico, pero queda sin resolver, la investigación privada o local, sobre la GIRH; especialmente, deja la inquietud sobre qué tanto los avances en la investigación sobre la GIRH han considerado la participación comunitaria, porque independiente de la cantidad de planes, programas o proyectos que se puedan generar desde las diferentes instancias, el aliento de vida de las propuestas, nace en la construcción colectiva con la comunidad (Posada A., 2014).
Para la para la WWAP (2017), la GIRH implica considerar también y en gran medida, el uso y la aceptación social de las aguas residuales, como parte del ciclo del recurso hídrico, porque conduce no solo a optimizar el recurso, sino a recuperarlo. Y en su estudio prospectivo, Rodríguez (2013) establece, como parte de las barreras para el futuro de la ciencia, la tecnología y la innovación al 2040, el escaso interés entre los políticos y empresarios para su desarrollo, la inexistencia de masa crítica de gestores e investigadores, así como de recursos financieros.

Este estudio dejó en evidencia que, en Colombia, solo una parte de la comunidad científica, es decir, los cinco institutos de investigación públicos de la Nación, trabajan directamente con los tomadores de decisiones. El resto de los investigadores de institutos o de universidades tienen limitada esta oportunidad, en los pocos y aislados proyectos, que se logran realizar, a través de convenios interinstitucionales, que resuelven preguntas de investigación, generan soluciones a problemáticas en asuntos puntuales, sobre la gestión del agua y en una temporalidad de corto o mediano plazo.

De aquí, se deduce que, para una adecuada política nacional de investigación sobre la GIRH, los tomadores de decisiones deben interactuar e integrarse con los diferentes grupos de investigación, de manera que las nuevas preguntas a los problemas pendientes de solucionar, se realicen en consenso y con una amplia gama de posibilidades, lo que corrobora la propuesta de Martin \& Justo (2015), sobre la necesidad de fortalecer los temas relativos a la gobernabilidad del recurso hídrico, detectando las fracturas entre los sectores y el territorio, para corregir los puntos críticos, donde no hay participación integral e integrada. Asunto que se complementa con la posición de Martínez \& Villalejo (2018), para quienes sigue pendiente la elaboración de una propuesta universalmente consensuada, de definición y de conceptualización sobre la GIRH.

En estas circunstancias, se hace un llamado a buscar la interacción entre las diferentes niveles, aristas, jerarquías, temas, grupos, ópticas y perspectivas, para avanzar en la GIRH, desde la complejidad y con un horizonte gradual de largo plazo, pero de inmediata acción. Desde la complejidad, debido a que este enfoque permite mayor amplitud y profundidad (Carrizosa, 2005). Y en un horizonte largo placista, porque se pueden considerar métodos que trascienden la generalidad y la abstracción, que impregna los modelos tradicionales (Vásquez, 2017).

En este camino, las ciencias ambientales ayudan a comprender y encontrar soluciones a la compleja crisis ambiental que vive la sociedad globalizada, en sus relaciones con la naturaleza (Aguirre, 2007). La generación de conocimiento, me- 
Cuadro 3. Avances regionales sobre la investigación en GIRH, en Colombia.

\begin{tabular}{|c|c|c|c|}
\hline GRUPO 1 & GRUPO 2 & GRUPO 3 & GRUPO 4 \\
\hline $\begin{array}{l}\text { - PORH } \\
\text { - PMAA } \\
\text { - POMCA } \\
\text { - Huella hídrica } \\
\text { - Módulos de consumo y } \\
\text { factores de vertimientos } \\
\text { - Reúso de aguas de pro- } \\
\text { ceso } \\
\text { - Zonas de recarga } \\
\text { - Macrozonificación } \\
\text { sísmica-amenazas } \\
\text { - Plan quebradas } \\
\text { - Monitoreo en aguas sub- } \\
\text { terráneas y superficiales } \\
\text { - Macroinvertebrados } \\
\text { - ERAS } \\
\text { - Caudal ambiental }\end{array}$ & $\begin{array}{l}\text { - Investigación directa no; } \\
\text { pero se han realizado } \\
\text { estudios de calidad, hi- } \\
\text { dráulicos, ecosistemas. } \\
\text { - Se ha participado en } \\
\text { procesos de generación } \\
\text { de conocimiento } \\
\text { - Alternativas para tratami- } \\
\text { ento de aguas de minas } \\
\text { sector Normandía } \\
\text { - La entidad no realiza } \\
\text { proyectos de investi- } \\
\text { gación. }\end{array}$ & $\begin{array}{l}\text { - No porque dentro del } \\
\text { presupuesto de las Cor- } \\
\text { poraciones y el ANLA no } \\
\text { hay fondos destinados } \\
\text { para tal actividad; no } \\
\text { obstante, si hay gestión } \\
\text { de conocimiento. } \\
\text { - Si desde lo técnico, so- } \\
\text { cial: Proyecto de conser- } \\
\text { vación de agua y suelo; } \\
\text { emprendimiento social } \\
\text { para la conservación } \\
\text { ambiental; educación en } \\
\text { GIRH para el desarrollo } \\
\text { sostenible; estudio de } \\
\text { amenaza vulnerabilidad } \\
\text { y riesgo; plan estratégico } \\
\text { de cultura del agua que } \\
\text { trabajan varios actores } \\
\text { del territorio desde niños } \\
\text { hasta juntas de acción } \\
\text { comunal; Observatorio } \\
\text { de conflictos ambientales } \\
\text { especialmente del agua; } \\
\text { Optimización de la PTAR. } \\
\text { - Centros de investigación } \\
\text { ambiental. } \\
\text { - Aguas subterráneas, } \\
\text { aguas termo minerales, } \\
\text { calidad del agua, para- } \\
\text { mos, regulación, ictiofau- } \\
\text { na/plancton, Fisicoquími- } \\
\text { cos, microbiológicos, } \\
\text { macro invertebrados, } \\
\text { servicios eco sistémicos, } \\
\text { modelación, coberturas. }\end{array}$ & 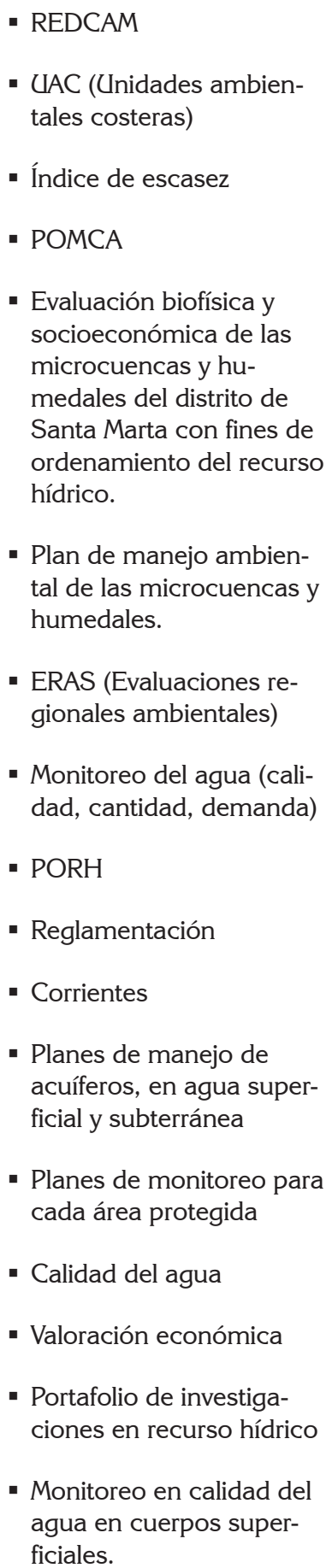 \\
\hline
\end{tabular}


Cuadro 4. Propuestas regionales para la investigación en GIRH, en Colombia.

\begin{tabular}{|c|c|c|c|}
\hline GRUPO 1 & GRUPO 2 & GRUPO 3 & GRUPO 4 \\
\hline $\begin{array}{l}\text { Interacción de acuífer- } \\
\text { os } \\
\text { Módulos de consumo } \\
\text { por sector } \\
\text { Abatimiento perma- } \\
\text { nente del nivel freático } \\
\text { Vulnerabilidad de } \\
\text { acuíferos } \\
\text { Metodologías integrales } \\
\text { de cálculo de oferta y } \\
\text { demanda } \\
\text { Estrategias de finan- } \\
\text { ciación nacional inter- } \\
\text { nacional para la GIRH } \\
\text { Parámetros de color en } \\
\text { la calidad del agua } \\
\text { Sistemas internos de } \\
\text { tratamiento de agua } \\
\text { residual } \\
\text { Determinación de } \\
\text { cantidad de agua } \\
\text { abatida por diferentes } \\
\text { usuarios } \\
\text { Investigaciones sobre } \\
\text { el avance de la mancha } \\
\text { de contaminación y } \\
\text { su impacto sobre el } \\
\text { acuífero } \\
\text { Determinación del } \\
\text { impacto del cambio de } \\
\text { cobertura vegetal sobre } \\
\text { la función de la regu- } \\
\text { lación hídrica y servi- } \\
\text { cios eco sistémicos. } \\
\text { Fortalecimiento Institu- } \\
\text { cional }\end{array}$ & $\begin{array}{l}\text { Cantidad } \\
\text { Calidad } \\
\text { Fuentes alternativas } \\
\text { Sedimentos } \\
\text { Hidráulica fluvial } \\
\text { Dinámica fluriomorfo- } \\
\text { logica } \\
\text { Carga contaminante en } \\
\text { red de drenaje } \\
\text { Conflictos por uso del } \\
\text { agua } \\
\text { Análisis prospectivo con } \\
\text { inclusión de fenómeno } \\
\text { de cambio climático y } \\
\text { variabilidad } \\
\text { Sistemas de medición } \\
\text { en ríos de montaña } \\
\text { Reabastecimiento de } \\
\text { información local y } \\
\text { propiciar un análisis } \\
\text { holístico } \\
\text { Cuantificación de im- } \\
\text { pactos antrópicos } \\
\text { Oferta y demanda en } \\
\text { escenarios de cambio } \\
\text { climático } \\
\text { Valoración integral del } \\
\text { recurso hídrico } \\
\text { Pago por servicios am- } \\
\text { bientales } \\
\text { Minería } \\
\text { Caudal ecológico } \\
\text { Gobernanza del agua }\end{array}$ & 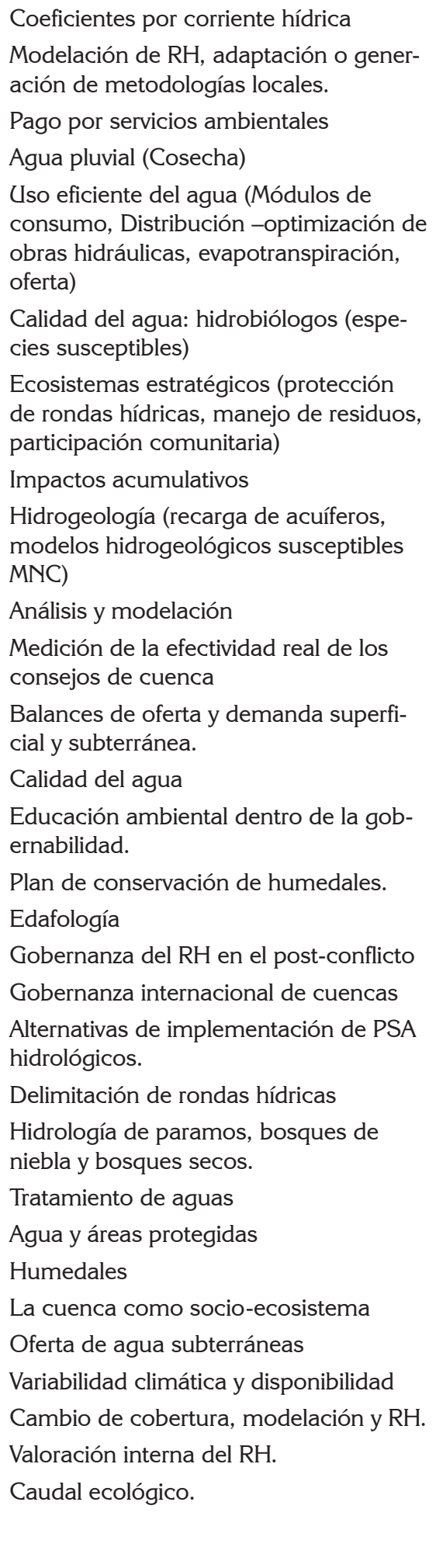 & 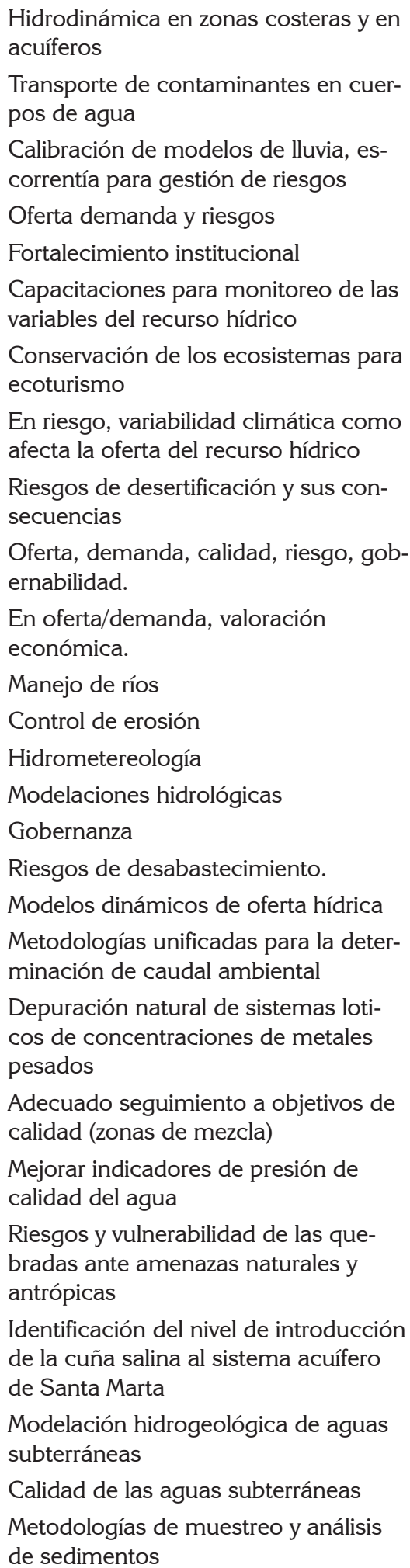 \\
\hline
\end{tabular}


diante la investigación ambiental, para tener una adecuada $\mathrm{GIRH}$, no solo es de interés y responsabilidad de una comunidad científica, si no de directrices institucionales y normativas nacionales, encaminadas al desarrollo y planeación de las regiones y, en general, del país, para atacar debilidades en cada equipo de trabajo y, de manera conjunta, entretejer la red necesaria, que no permita dejar escapar de nuestras vidas, al preciado recurso hídrico.

Finalmente, es necesario continuar construyendo conocimiento en líneas o áreas temáticas, que permitan abordar la gestión integral del recurso hídrico, como eje articulador y funcional, para el desarrollo de la vida misma, fortaleciendo el relacionamiento interinstitucional y creando alianzas importantes, entre los diferentes sectores del país, que conlleven a estrategias financieras, técnicas, de fortalecimiento, para continuar procesos investigativos de gran impacto, en pro del desarrollo sostenible del país y del mundo.

Agradecimiento: Las autoras agradecen a la U.D.C.A y al Ministerio de Ambiente y Desarrollo Sostenible, en particular a la Dirección de Gestión Integral del Recurso Hídrico, por apoyar el proyecto de investigación "Líneas de investigación como base para la gestión integral del recurso hídrico en Colombia" que, en gran parte, permitió la elaboración de este manuscrito. Conflicto de Intereses: El manuscrito fue preparado y revisado por las autoras, quienes declaran ser las que originaron el artículo y no presentan conflicto de intereses.

\section{REFERENCIAS}

1. AGUDELO C., N. 2004. Las líneas de investigación y la formación de investigadores: una mirada desde la administración y sus procesos formativos. Revista Electrónica de la Red de Investigación Educativa (Colombia). 1(1):13. Disponible desde Internet en: http://revista.iered.org/v1n1/pdf/ncagudelo.pdf (con acceso el 19/09/2017).

2. AGUIRRE M., D. 2007. Educación superior colombiana y medio ambiente. Facultad de Ciencias Ambientales UTP. Compilado en libro: Las Ciencias Ambientales: Una Nueva Área Del Conocimiento RCFA (Colombia). Primera edición. p.85-99.

3. ANDRADE, P.A.; LE BLAS, F.N. 2004. Lineamientos para la aplicación del enfoque ecosistémico a la gestión integral del recurso hídrico. Programa de las Naciones Unidas para el Medio Ambiente - PNUMA, Oficina Regional para América Latina y el Caribe, Red de Formación Ambiental (México).111p.
4. CARRIZOSA U., J. 2005. Notas Alrededor de la Investigación Ambiental. Gestión y Ambiente (Colombia). 8(2):7-23.

5. CONGRESO DE COLOMBIA. 1990. Ley No. 29 de 1990. Por la cual se dictan disposiciones para el fomento de la Investigación Científica y el Desarrollo Tecnológico. Diario oficial. Disponible desde Internet en: http://www.alcaldiabogota.gov.co/sisjur/normas/ Norma1.jsp?i=254 (con acceso el 1/09/2017).

6. DE BARRERA, J.H. 2010. Líneas de investigación y gerencia del conocimiento: Premisas de la cultura de investigación. Trilogía Ciencia Tecnología Sociedad (Colombia). 2:83-92.

7. GLOBAL WATER PARNERTSHIP -GWP-. 2000. Manejo integrado de recursos hídricos. Papers $\mathrm{N}^{\circ} 4$ : Comité de Consejo Técnico (TAC) de la Asociación Mundial para el Agua (Suecia). Disponible desde Internet en: https://www.gwp.org/globalassets/global/toolbox/ publications/background-papers/04-integratedwater-resources-management-2000-spanish.pdf (con acceso el 01/02/2018).

8. GUHL N., E.; LEYVA, P. 2015. La gestión ambiental en Colombia: 1994-2014 ¿ ¿Un esfuerzo insostenible? FESCOL. Foro Nacional Ambiental. QUINAXI. 224p.

9. HASSAN, F. 2011. Water History for our times. United Nations educational, scientific and Cultural organization (UNESCO). International Hydrological Programme (France). 122p.

10. IVANKOVICH G., C.; ARAYA Q., Y. 2011. "Grupos focales": Técnica de investigación cualitativa en investigación de mercados. Ciencias Económicas. 2011. Centro Nacional de Ciencia y Tecnología de Alimentos (Costa Rica). 29(1):545-554.

11. MARTIN, L.; JUSTO, J. B. 2015. Análisis, prevención y resolución de conflictos por el agua en América Latina y el Caribe. Serie CEPAL 71: recursos naturales e infraestructura. Naciones Unidas. (Chile). 62p.

12. MARTÍNEZ V., Y.; VILLALEJO G., V.M. 2018. La gestión integrada de los recursos hídricos: una necesidad de estos tiempos. Ing. Hidráulica y Ambiental 39(1):5872.

13. MARTINEZ-S., P.; ALDAYA, M.M.; LLAMAS, R. 2014. Integrated Water Resources Management in the 21st Century: Revisiting the Paradigm. Taylor \& Francis Group (London-UK). Chapter 2. p.17-36. 
14. MINISTERIO DE AMBIENTE, VIVIENDA Y DESARROLLO TERRITORIAL -MAVDT-; INSTITUTO PARA EL DESARROLLO SOSTENIBLE -QUINAXI-. 2007. Plan Estrategico Nacional de Investigación Ambiental, PENIA. Institutos de investigación adscritos y vinculados al MAVDT (Colombia). 79p. Disponible desde Internet en: http://www.humboldt. org.co/images/documentos/pdf/documentos/penia. pdf (con acceso el 5/08/2017).

15. MINISTERIO DE AMBIENTE, VIVIENDA Y DESARROLLO TERRITORIAL, MAVDT. 2010. Politica Nacional para la Gestión Integral del Recurso Hidrico. Grupo de Recursos Hídricos (Colombia).124p.

16. PAHL-WOSTL, C. 2007. The implications of complexity for integrated resources management. Environmental Modelling \& Software (Germany). 22:561-569. https://doi.org/10.1016/j.envsoft.2005.12.024

17. POSADA A., A. 2014. De la planeación a la gestión ambiental colectiva: posibilidades en Bogotá-Colombia. Rev. U.D.C.A Act \& Div. Cient. 17(1):257-264.

18. REPÚBLICA DE COLOMBIA. 2001. Politica Nacional de Investigación Ambiental. 38p. Disponible desde Internet en: http://oab.ambientebogota.gov.co/ es/con-la-comunidad/ES/politica-nacional-deinvestigacion-ambiental (con acceso el 18/09/2017).

19. RODRÍGUEZ F., J.J. 2013. Diseño prospectivo de escenarios para la ciencia, tecnología e innovación al 2040. Industrial Data (Perú). 16(2):92-105. https:// doi.org/10.15381/idata.v16i2.11927

20. SÁENZ Z., O. 2003. La investigación sobre ambiente y hábitat. Organización institucional y olíticas recientes. Ciencia y Tecnologia (Colombia ). 21(2):5-15.
21. VÁSQUEZ, J.A. 2017. Integrated water resources management: from a complexity theory perspective. WIT transactions on ecology and the environment. Water and Society (England). 216(4):1-11. https:// doi.org/10.2495/WS170011

22. UNITED NATIONS WORLD WATER ASSESSMENT PROGRAMME -WWAP-. 2017. Wastewater: The Untapped Resource. The United Nations World Water Development Report- UNESCO (Paris). 198p.

23. UNITED NATIONS WORLD WATER ASSESSMENT PROGRAMME -WWAP-. 2018. Informe Mundial de las Naciones Unidas sobre el Desarrollo de los Recursos Hídricos 2018: Soluciones basadas en la naturaleza para la gestión del agua. UNESCO (Paris). 168 .

24. UN WATER. 2008. Status report on integrated water resources management and water efficiency plans. Prepared for the 16th session of the commission on sustainable development (New York). 48p.

25. WINZ, I.; BRIERLEY, G.; TROWSDALE, S. 2009. The use of system dynamics simulation in water resources management. Water Resour Manag (Europa). 23(7):1301-1323. https://doi.org/10.1007/s11269008-9328-7

Recibido: Mayo 6 de 2018

Aceptado: Noviembre 10 de 2018

\section{Cómo citar:}

Hernández Pasichana, S.M.; Posada Arrubla, A. 2018. Avances de la investigación sobre la gestión integral del recurso hídrico en Colombia. Rev. U.D.C.A Act. \& Div. Cient. 21(2):553-563. https://doi.org/10.31910/rudca.v21.n2.2018.1079 